safety but challenging in our field, and not without barriers. The purpose of this analysis was to determine how often medications errors get communicated to patients and/or families and to identify the barriers perceived by healthcare providers to disclose these errors.

Methods A survey was distributed to the critical care team. The questions were answered anonymously, with the only identifier being their position in the critical care team.

Results A 76\% response was obtained, eight attendings (44.4\%), 5 nurses-RNs (27.8\%), 3 nurse practitioners- NPs (16.7\%), and 3 fellows (16.7\%) returned the survey. The group that 'always' reported communication of medicine errors was the attendings (42\%), followed by RNs (40\%), NPs (25\%), and the fellows $(0 \%)$ (Figure 1). The most often perceived obstacle to communicating was family not being available (Figure 2).

Conclusion This analysis demonstrated that communication of medication errors does not happen consistently. In addition, the most common obstacle identified was the absence of family when the event occurs. This is most likely a challenge that is more unique to the paediatric population. The culture of open communication is critical in creating a safer medical environment; therefore, it is a skill that must be implemented into the medical education.

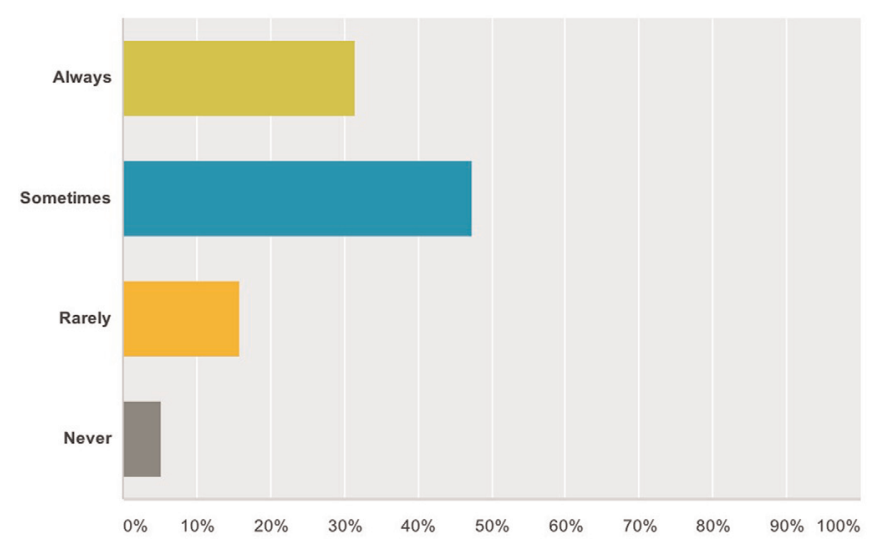

Abstract P0-0332 Figure 1 Frequency of communication of medication errors to the patient/family

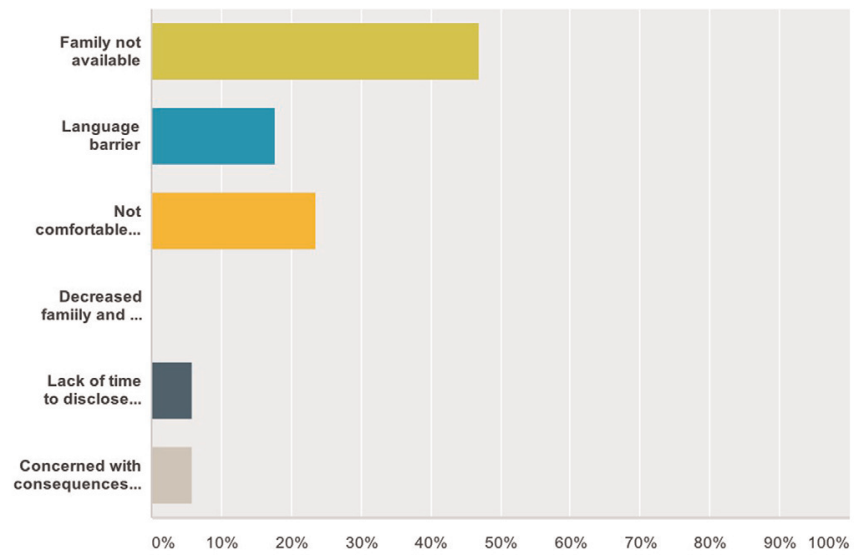

Abstract P0-0332 Figure 2 Barriers encountered in communicating medication errors to a patient/family

\section{PO-0333 MUSCLE THICKNESS ULTRASONOGRAPHY IN CRITICALLY ILL CHILDREN: ANALYSIS OF ACCURACY}

${ }^{1} \mathrm{~T}$ Fivez, ${ }^{1} \mathrm{~A}$ Hendrickx, ${ }^{2} \mathrm{~F}$ Claus, ${ }^{1} \mathrm{~L}$ Desmet, ${ }^{1} \mathrm{D}$ Vlasselaers, $1,3 \mathrm{~T}$ Van Herpe, ${ }^{1} \mathrm{G}$ Van den Berghe, 'D Mesotten. ' $\mathrm{KU}$ Leuven, Dept Intensive Care Medicine, Leuven, Belgium; ${ }^{2}$ OLVZ-Aalst, Radiology Unit, Belgium; ${ }^{3} \mathrm{KU}$ Leuven, Dept Electrical EngineeringESAT-SCD/iMinds Future Health

\subsection{6/archdischild-2014-307384.982}

Background and aims Critical illness results in muscle wasting, typically within the first week of admission. However, its quantification is difficult. Ultrasonographical evaluation has already been tested in adult patients. Here we aim to assess of the accuracy of this methodology in critically ill children.

Methods Two independent investigators made ultrasonographical assessments of muscle thickness. A linear array commercial real time ultrasound scanner (Vivid S6) was used with a $12-\mathrm{MHz}$ transducer. The transducer was placed perpendicularly to the long axis of the tight on three fifths of the distance from the anterior superior iliac spine to the superior patellar border. An excess of contact gel was applied to minimise image distortion. During the first week of critical illness we hypothesised a reduction in muscle size of $30 \%$ based on literature of critically ill adult patients.

Results A group of 43 patients (newborns to 4-years old children) were included in the study. The average muscle thickness was $1.67 \mathrm{~cm}$ (SD: $0.52 \mathrm{~cm}$ ). Hence 30\% reduction would equal $0.50 \mathrm{~cm}$. The intra-observer variability, as expressed by the limits of agreement $( \pm 1.96 \mathrm{SD}$, containing $95 \%$ of the samples for normally distributed samples), was $0.61 \mathrm{~cm}$ for operator 1 and $0.83 \mathrm{~cm}$ for operator 2 . Both distributions are visualised in a Bland-Altman plot in Figure 1. Combining both operator data revealed an observer variability of $0.72 \mathrm{~cm}$. The median absolute inter-observer variability was $0.085 \mathrm{~cm}$ [IQR $0.040-0.20 \mathrm{~cm}$ ]. Conclusions Although the inter-observer variability among the two operators is acceptable, the intra-observer variability may be too large with respect to the limited expected muscle reduction. Therefore, ultrasonographical analyses of muscle wasting need more standardisation for use in critically ill children.

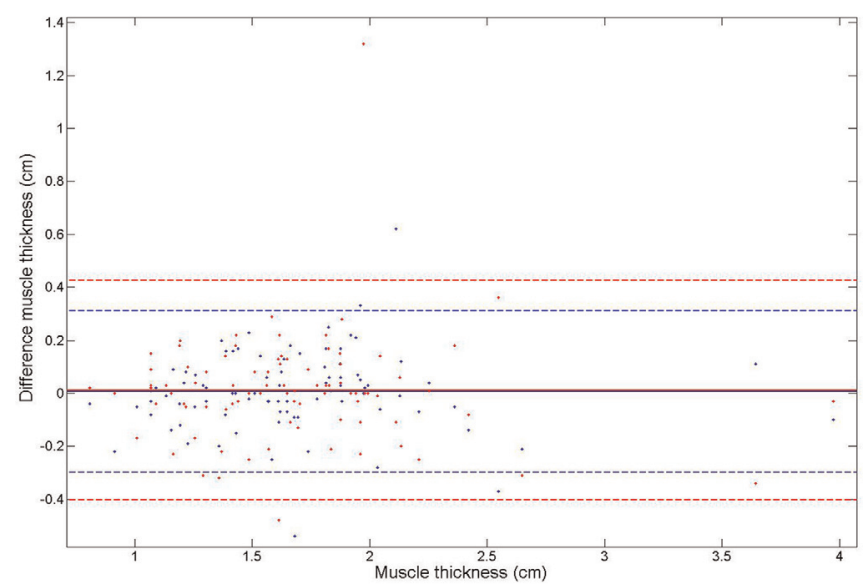

Abstract P0-0333 Figure 1 